\title{
Tooth Auto-transplantation: An Alternative Treatment
}

\author{
Jawaad Ahmed Asif ${ }^{1)}$, Tahir Yusuf Noorani ${ }^{2)}$ and \\ Mohammad Khursheed Alam ${ }^{3)}$ \\ ${ }^{1)}$ Oral and Maxillofacial Surgery Unit, School of Dental Sciences, Universiti Sains Malaysia, \\ 16150 Kubang Kerian, Kelantan, Malaysia \\ ${ }^{2)}$ Conservative Dentistry Unit, School of Dental Sciences, Universiti Sains Malaysia, \\ 16150 Kubang Kerian, Kelantan, Malaysia \\ ${ }^{3)}$ Orthodontic Department, College of Dentistry, Al Jouf University, \\ Sakaka 72388, Kingdom of Saudi Arabia
}

Received 6 May, 2016/Accepted for publication 7 June, 2016

\begin{abstract}
Auto-transplantation is the repositioning of an autogenously erupted, partially erupted, or unerupted tooth from one site to another in the same individual. The purpose of this case report is to describe the auto-transplantation of the mandibular third molars as an alternative means of replacing second molars requiring extraction. An orthopantomogram revealed horizontal impaction of teeth \#37 and 47 and mesio-angular impaction of teeth \#38 and 48. Cone beam computed tomography revealed that the roots of \#37 and 47 were in close proximity to the inferior alveolar canal bilaterally. As atraumatic and complete removal of these two teeth was not possible, \#38 and 48 were chosen as the donor teeth instead. The procedure was carried out under general anesthesia. Teeth \#38 and 48 were extracted atraumatically; teeth \#37 and 47 were pulled out by sectioning and transplant sites prepared. Teeth \#38 and 48 were transplanted to the sites vacated by teeth \#37 and 47, respectively. Endodontic treatment of teeth \#38 and 48 was completed within 1 month following transplantation. Both clinical and radiographic outcomes were considered satisfactory at 1 year postoperatively. Bone healing was observed around the roots of teeth \#38 and 48. In conclusion, although auto-transplantation is not very common in general dental practice, we believe that it offers a clinically and economically viable alternative to other complicated prosthetic and dental implant treatments.
\end{abstract}

Key words: Auto-transplantation — Cone beam computed tomography (CBCT) Orthopantomogram (OPG) - Xenograft

\section{Introduction}

Auto-transplantation is the repositioning of an autogenously erupted, partially erupted, or unerupted tooth from one site to another in the same individual. It is a viable treatment 
option in replacing a missing tooth or one that requires extraction due to caries, periodontal disease, or some other reason ${ }^{13)}$. It involves atraumatic removal of a donor tooth, creation of a socket at the recipient site, and re-implantation of the tooth into the new position. Thus aesthetics and mastication can be improved while maintaining arch space and preserving the volume and morphology of the alveolar bone ${ }^{6)}$. It can also be performed as a single-step surgical procedure ${ }^{2}$. Therefore, with careful case selection, auto-transplantation can serve as a fast, feasible, and economic treatment option, especially in young patients ${ }^{1)}$.

A number of factors influence the success rate of auto-transplantation, including the developmental stage of the root; the tooth morphology; the surgical procedure selected; the extra-oral time; the shape/site of the recipient socket; the vascularity of the recipient bed; and the viability of the periodontal ligament cells ${ }^{9}$. Avulsed teeth recover optimal function and esthetics after re-implantation under ideal conditions, and similar healing patterns can be expected in auto-transplantation of teeth. While dental implantation offers the most desirable treatment option for a missing tooth, it cannot always be applied due to the youth of the patient or socio-economic reasons. Auto-transplantation offers an alternative in such cases $^{7}$.

The purpose of this case report is to describe the auto-transplantation of the mandibular third molars as an alternative means of replacing second molars requiring extraction.

\section{Case Report}

The patient was a healthy 24-year-old woman who presented at the Department of Oral and Maxillofacial Surgery at Universiti Sains Malaysia with bilateral impacted mandibular third molars. She had been referred to us by her orthodontist with a request for removal of the impacted teeth. Clinically, the patient's oral hygiene was good; crowding was observed in the mandibular anterior denti-

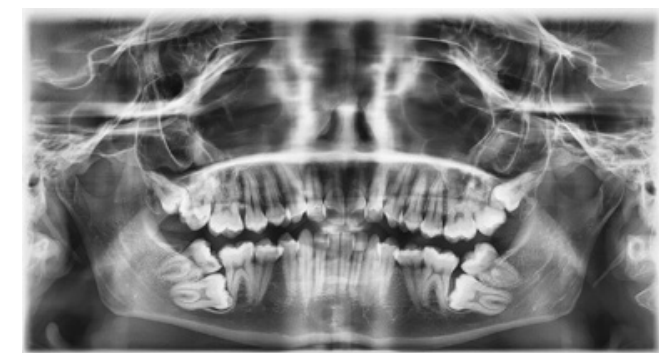

Fig. 1 Pre-treatment orthopantomogram (OPG) showing impacted teeth \#18, 28, 37, 38, 47, and 48

tion. The mandibular second molars (\#37 and 47) were not visible clinically, while the distal portion of \#38 and 48 was only partially so. An orthopantomogram (OPG) was obtained to evaluate the level of impaction of all third molars. Radiograph examination revealed horizontal impaction of \#37 and 47 and mesio-angular impaction of \#38 and 48 (Fig. $1)$. Both the maxillary third molars were also found to be impacted. The impacted mandibular second molars were in a close proximity to the inferior alveolar canal (IAC) bilaterally. Cone beam computed tomography was used to determine the proximity of the mandibular second molars to the IAC. The results revealed that the roots of the mandibular left second molar were closer to the IAC than those of its right counterpart (Figs. 2 and 3). Due to this proximity, these teeth were judged as unsuitable for transplantation. Indeed, atraumatic and complete removal of these teeth would have required excising a considerable amount of bone and might have also traumatized the underlying nerve bundle. Therefore, \#38 and 48 were chosen as the donor teeth. Teeth \#37 and 47 were to be removed by sectioning. Additionally, bone thickness was analyzed using Planmeca Romexis version 2.9.2 software. The total bucco-lingual width on the right side involving \#48 and 47 was $17 \mathrm{~mm}$. Buccal bone thickness was $1.5 \mathrm{~mm}$, while lingual bone thickness was $3 \mathrm{~mm}$. On the left side, the total buccolingual width involving \#38 and 37 was $18 \mathrm{~mm}$. Here, buccal bone thickness was $3.2 \mathrm{~mm}$, while lingual bone thickness was $1.6 \mathrm{~mm}$. 

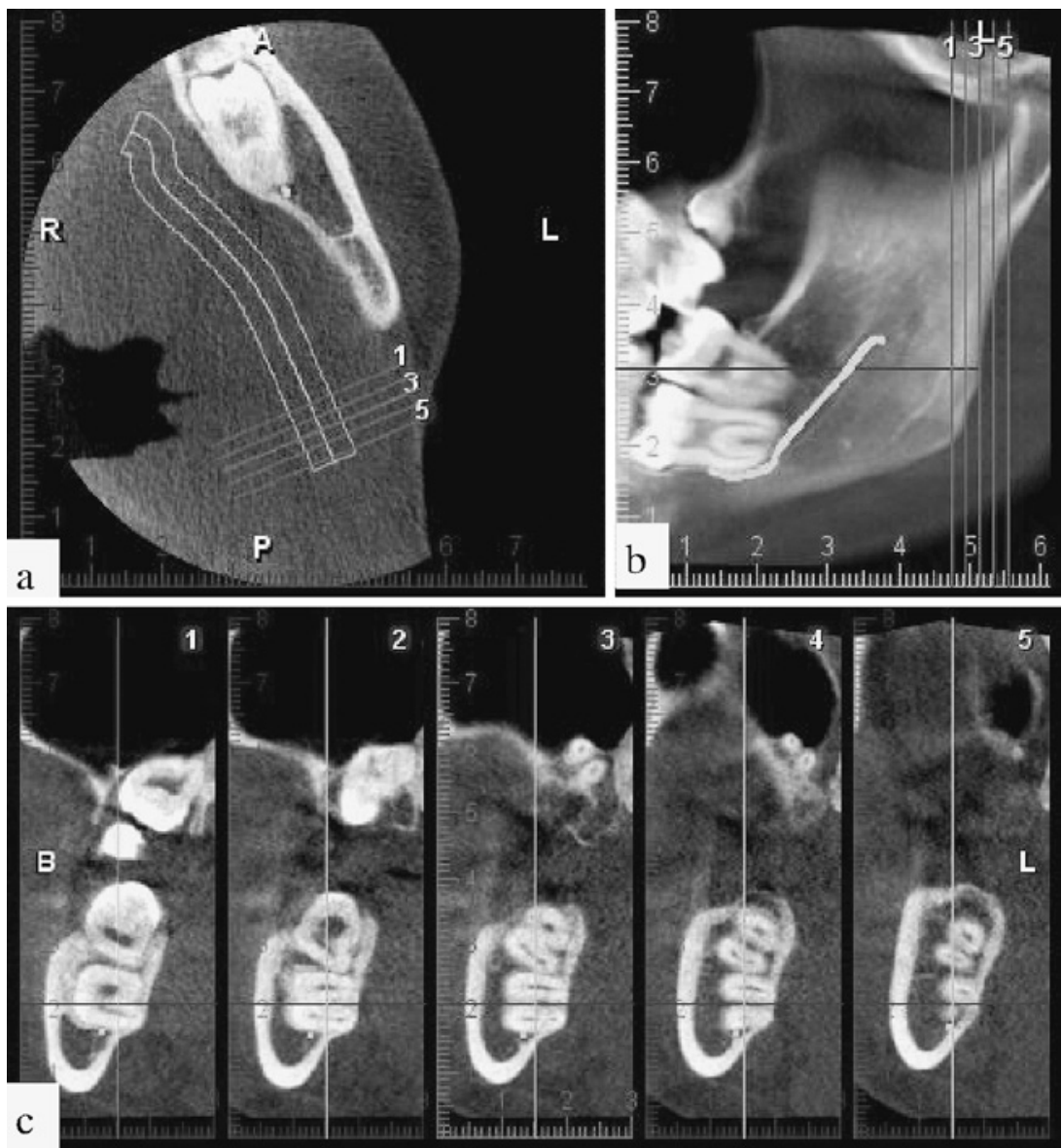

Fig. 2 Cone beam computed tomograph (CBCT) showing proximity of mandibular left second molar to inferior alveolar canal (IAC)

a) axial section, b) saggital section, c) coronal sectional slices.

These measurements indicated that there was adequate bucco-lingual bone width available at the transplant sites. After taking a complete medical history and explaining the risks and benefits of the procedure to the patient, informed consent was obtained.

The surgical procedure was carried out under general anesthesia. Thorough intraoral irrigation with chlorhexidine $0.2 \%$ (HUSM pharmacy, Malaysia) was performed to decrease the microbial load. Local anesthetic was delivered to the bilateral inferior alveolar nerves by block technique using $2 \%$ Scandonest Special (Septodont, USA) and 2 cartridges $(2.2 \mathrm{ml})$ of adrenaline $(1: 100,000)$.
Triangular flap design provided excellent access to the surgical site. Tooth \#38 was removed atraumatically by removing the minimum amount of distal bone; the tooth was then stored in pasteurized milk while \#37 was being removed (Fig. 4a). Removal of tooth \#47 took 7-9 minutes; the socket was then debrided by irrigation with sterile saline solution to prepare the recipient site (Fig. 4b). The underlying neuro-vascular bundle was intact. Donor tooth \#38 was carefully handled and placed into the prepared transplant site (Fig. 4c). It was positioned below the occlusal level and stabilized using $0.4-\mathrm{mm}$ thick stainless steel wire and composite resin 

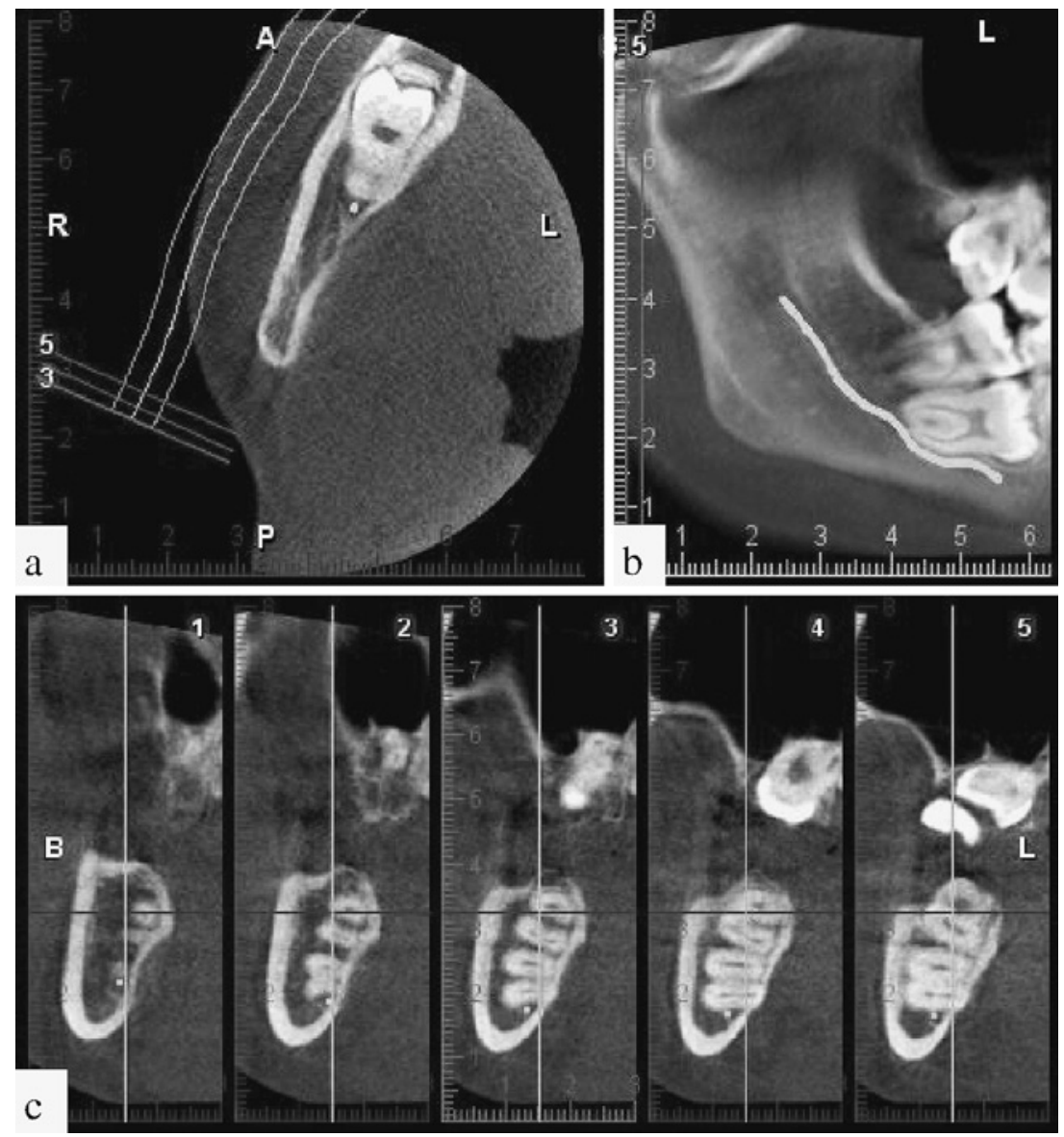

Fig. 3 CBCT showing proximity of mandibular right second molar to IAC a) axial section, b) saggital section, c) coronal sectional slices.

(Filtek Z350, 3M ESPE, USA) (Fig. 4d). The exposed root area was covered with freezedried bovine bone graft (OsteoLEMB, National Tissue Bank, Universiti Sains Malaysia). The surrounding soft tissue was then repositioned and sutured using Safil 4/0 (Aesculap, USA) absorbable suture. A similar procedure was carried out on the right side, where tooth \#48 was transplanted into the site vacated by tooth \#47. The maxillary third molars were also surgically removed after completion of transplantation. Postoperative recovery was uneventful and the patient was discharged within 24 hours after surgery. The patient was instructed to perform daily mouth rinsing with $0.12 \%$ chlorhexidine gluconate,
3 times a day for 7 days. At a follow-up visit 1 week later, healing around the surgical area was uneventful (Fig. 5). No associated paresthesia was observed on either side of the lower area of the face. An OPG was obtained to reassess the position of the transplanted teeth. The position and level of the transplanted teeth were satisfactory (Fig. 6).

Composite wire splints were removed on the 15th day postoperatively. Resin material together with the wire was cut away from the tooth surface using a flame-shaped diamond finishing bur at high speed with an air/water spray. The tooth surfaces were then polished using a superfine aluminum oxide disc and mobility assessed. Both teeth \#38 and 48 dem- 

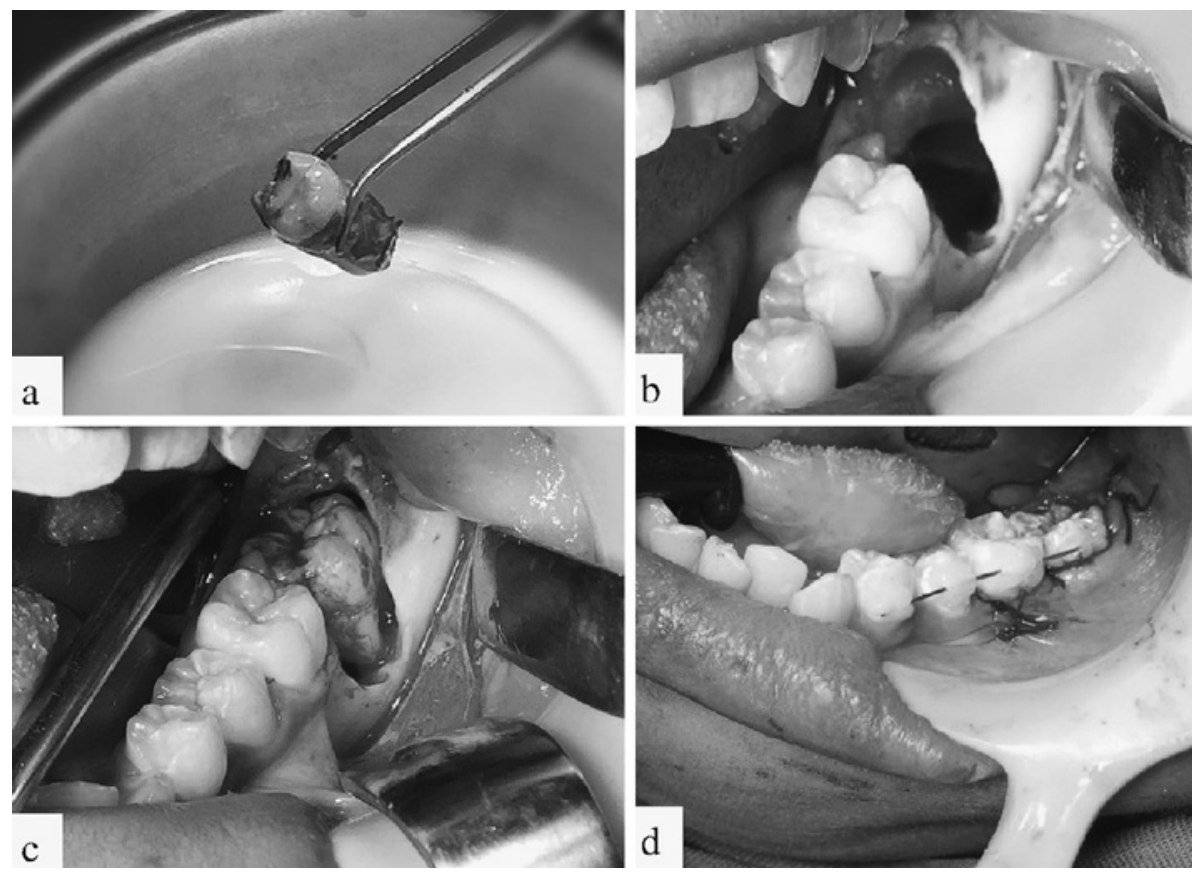

Fig. 4 Auto-transplantation of tooth \#38

a) Donor tooth stored in milk following extraction, prior to transplantation, b) Prepared transplant site, c) Tooth \#38 in place of \#37, d) Tooth stabilized using composite-wire splint.
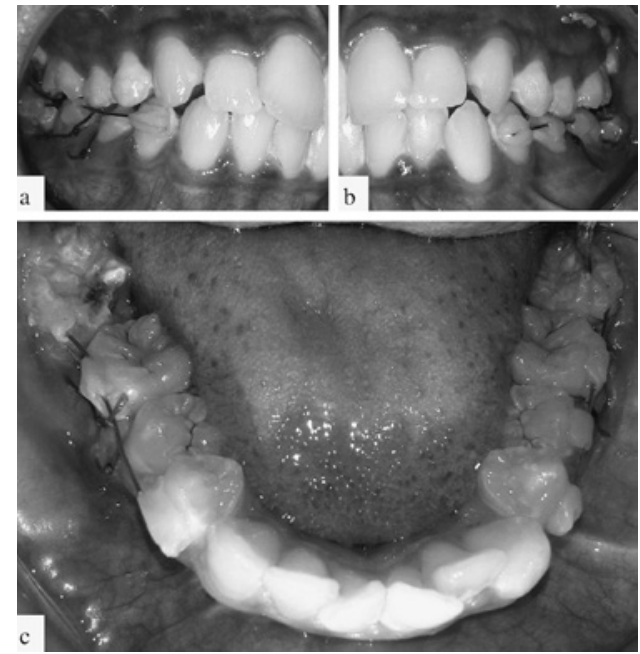

Fig. 5 Intra-oral photographs obtained at 1 week postoperatively; note sutures have not yet resorbed completely

a) right lateral view, b) left lateral view, c) lower occlusal view.

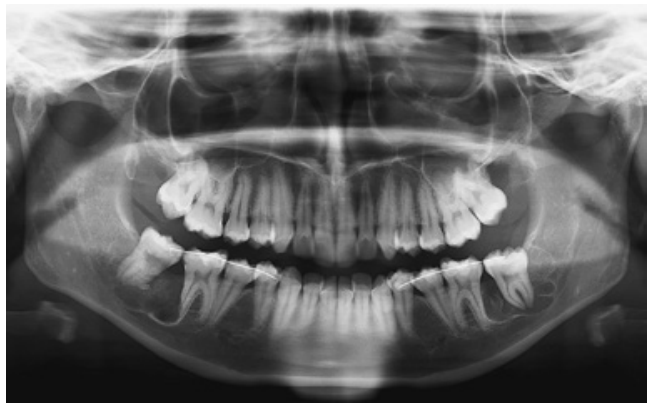

Fig. 6 OPG obtained at 1 week following auto-transplantation of teeth \#38 and 48 to replace teeth \#37 and 47, respectively

onstrated normal physiologic tooth mobility. Endodontic treatment for teeth \#38 and 48 was then carried out (Fig. 7). Tooth \#38 was isolated and an access cavity prepared. Shaping was performed with Protaper universal rotary files (Dentsply Tulsa Dental, USA), while irrigation involved alternate application 

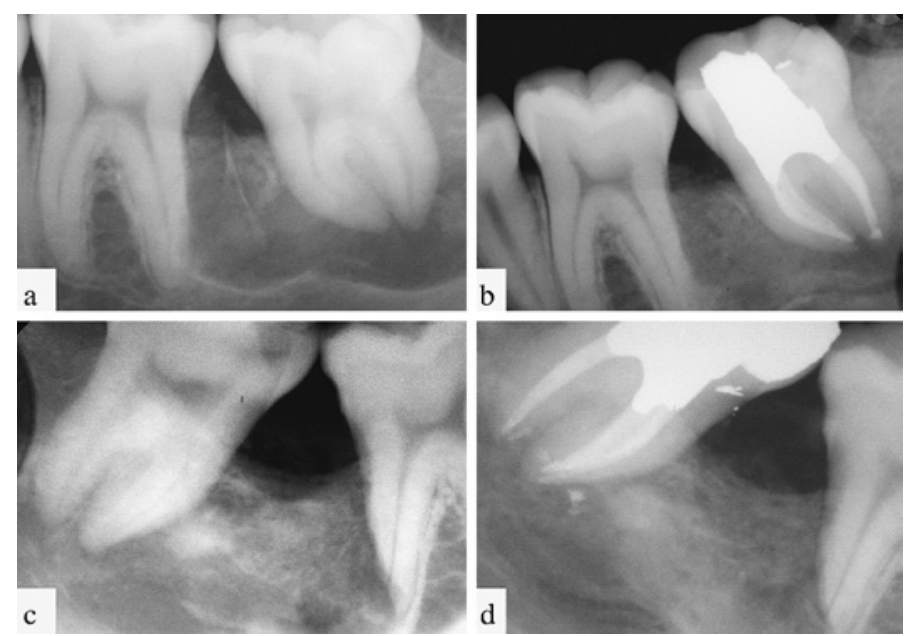

Fig. 7 Periapical radiographs showing endodontic treatment of teeth \#38 and 48

a) Pre-op radiograph of \#38, b) Post-op radiograph of \#38, c) Pre-op radiograph of \#48, d) Post-op radiograph of \#48.

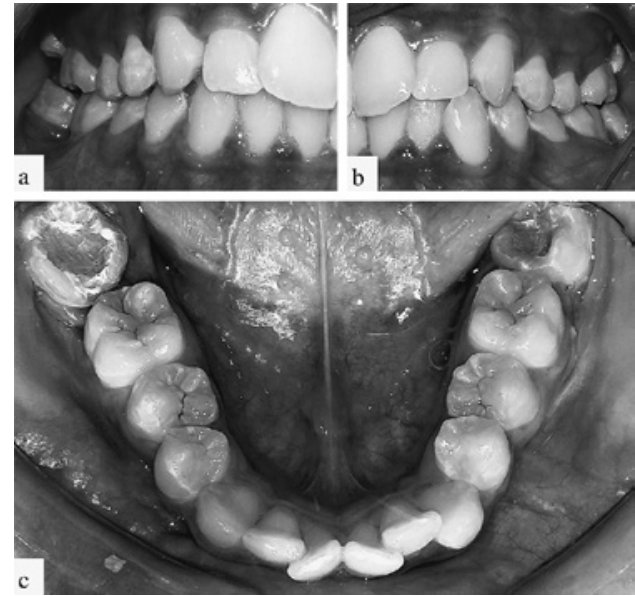

Fig. 8 Intra-oral photographs following endodontic treatment and restoration of tooth \#38 and 48, 1 month after surgery

a) right lateral view, b) left lateral view, c) lower occlusal view.

of $2.5 \%$ sodium hypochlorite (HUSM Pharmacy, Malaysia) and 17\% EDTA (Ethylene diamine tetra-acetic acid) (Dentonics Inc., USA). The canals were obturated with gutta percha using warm vertical condensation technique and AH Plus (Dentsply Maillefer,

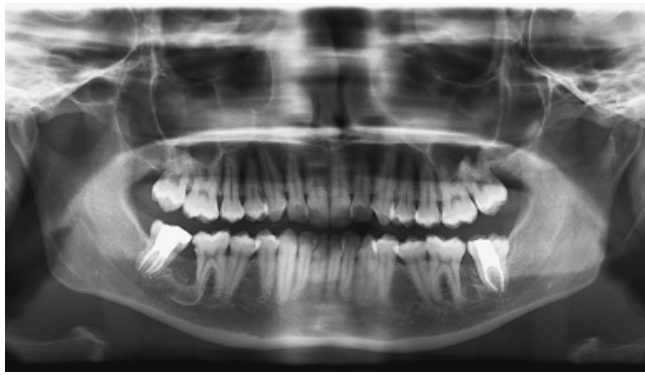

Fig. 9 OPG obtained at 1 year following auto-transplantation

Switzerland) resin-based root canal sealer. Following endodontic treatment, coronal access cavity was restored with a Nayyar core amalgam restoration. Endodontic treatment and amalgam restoration were also performed on tooth \#48 in a similar fashion. Endodontic treatment for both \#38 and 48 was completed within the first month following transplantation. The patient was reviewed at 1 (Fig. 8), 3, and 6 months, and 1 year following completion of treatment. A review OPG was obtained at 1 year after completion of treatment (Fig. 9 ). Bone healing was observed around the roots of teeth \#38 and 48. Both clinical and 
radiographic outcomes were considered satisfactory after 1 year.

\section{Discussion}

Auto-transplantation offers an alternative treatment option in replacing a missing tooth or one requiring extraction. Some studies have reported a survival rate of up to $90 \%$ over 5 years with this procedure ${ }^{5,13,15,16)}$. For a successful outcome, however, auto-transplantation requires meticulous candidate selection and procedural planning. The selection criteria include factors such as general health, psychological behavior, willingness to undergo the procedure and, most importantly, oral hygiene status.

Both the donor tooth and recipient site should be examined with the utmost care to ensure compatibility and fit. Adequate bone support with sufficient attached keratinized tissue to allow tooth stabilization are required at the recipient site, which must also be free of infection and/or inflammation ${ }^{3)}$. Inadequate bucco-lingual bone width here or excessive preparation may result in resorption of the alveolar ridge and loss of buccal bone coverage, which may greatly affect periodontal healing following transplantation. One study concluded that lack of buccal bone plate was the only significant predictor of transplant failure ${ }^{5)}$.

Following auto-transplantation of teeth with complete root formation, endodontic treatment has to be carried out to prevent pulp infection from spreading to the periapical area and subsequent inflammatory root resorption ${ }^{13}$. This is necessary because revascularization of pulp is not expected to occur in teeth with completely formed roots ${ }^{4,12,13)}$. In the present case, endodontic treatment could have been carried out extra-orally before transplantation. However, this was decided against as this would have increased the time span between extraction and transplantation. The longer the time lapse between these two points, the more negative the effect on the prognosis. The prognosis of teeth that are auto-transplanted is best when the time between removal of the tooth and re-implantation is shortest ${ }^{14)}$. Moreover, there is always the risk of damaging the periodontal ligament fibers and cells when performing endodontic treatment extra-orally. Another factor that may affect the prognosis is the temporary storage medium, with pasteurized milk being considered the most suitable after cell and tissue culture solutions such as Hank's balanced salt solution $(\mathrm{HBSS})^{10)}$. The advantages of milk over water or saline include its physiologically compatible $\mathrm{pH}$, osmolality with cells attached to the root surface, and presence of nutrients and growth factors. As HBSS was not available, pasteurized milk was used in the present case.

Following endodontic treatment, both teeth \#38 and 48 were restored with amalgam restoration. No further restorative treatment was planned for \#38, as only a limited amount of tooth structure was cut to access the root canals for endodontic treatment of this tooth. However, the mesial marginal ridge was sacrificed in tooth \#48 due to the presence of caries on the mesio-occlusal aspect, which required the placement of a large mesioocclusal amalgam restoration. Hence, restoration of tooth \#48 with a full coverage coronal restoration was advisable ${ }^{11)}$. However, tooth \#48 was not presently occluding with the opposing tooth. Hence, its restoration with a crown was delayed until completion of orthodontic treatment. We should mention that this tooth was purposely kept out of occlusal contact during transplantation to prevent occlusal forces from acting on the tooth, which might have interfered with the healing of the periodontium following transplantation ${ }^{8)}$.

\section{Conclusion}

Although not very common in general dental practice, we believe that auto-transplantation offers a clinically and economically viable alternative to other complex prosthetic and dental implant treatments. Careful case selection, atraumatic surgical technique, and 
meticulous adherence to the biologic principles of healing can yield satisfactory outcomes.

\section{References}

1) Azevedo PC, Moura CCG, Zanetta-Barbosa D, Bernadineli N (2007) Time of endodontic treatment in autogenic transplants of mature teeth: histological study in dogs. Oral Surg Oral Med Oral Pathol Oral Radiol Endod 104: 287-293.

2) Bae JH, Choi YH, Cho BH, Kim YK, Kim SG (2010) Autotransplantation of teeth with complete root formation: a case series. J Endod 36: 1422-1426.

3) Clokie CM, Yau DM, Chano L (2001) Autogenous tooth transplantation: An alternative to dental implant placement? J Can Dent Assoc 67:92-96.

4) Hasselgren G, Larsson Å, Rundquist L (1977) Pulpal status after autogenous transplantation of fully developed maxillary canines. Oral Surg Oral Med Oral Pathol 44:106-112.

5) Mejàre B, Wannfors K, Jansson L (2004) A prospective study on transplantation of third molars with complete root formation. Oral Surg Oral Med Oral Pathol Oral Radiol Endod 97:231-238.

6) Mendes RA, Rocha G (2004) Mandibular third molar autotransplantation - literature review with clinical cases. J Can Dent Assoc 70: 761-766.

7) Nimčenko T, Omerca G, Varinauskas V, Bramanti E, Signorino F, Cicciù M (2013) Tooth auto-transplantation as an alternative treatment option: a literature review. Dent Res J (Isfahan) 10:1-6.

8) Park JH, Tai K, Hayashi D (2010) Tooth autotransplantation as a treatment option: a review. J Clin Pediatr Dent 35:129-135.

9) Patel S, Fanshawe T, Bister D, Cobourne MT
(2011) Survival and success of maxillary canine autotransplantation: a retrospective investigation. Eur J Orthod 33:298-304.

10) Poi WR, Sonoda CK, Martins CM, Melo ME, Pellizzer EP, De Mendonca MR, Panzarini SR (2013) Storage media for avulsed teeth: a literature review. Braz Dent J 24:437-445.

11) Sivers J, Johnson W (1992) Restoration of endodontically treated teeth. Dent Clin North Am 36:631-650.

12) Skoglund A (1981) Pulpal changes in replanted and autotransplanted apicoectomized mature teeth of dogs. Int J Oral Surg 10: 111-121.

13) Sugai T, Yoshizawa M, Kobayashi T, Ono K, Takagi R, Kitamura N, Okiji T, Saito C (2010) Clinical study on prognostic factors for autotransplantation of teeth with complete root formation. Int J Oral Maxillofac Surg 39: 1193-1203.

14) Tsukiboshi M (2002) Autotransplantation of teeth: Requirements for predictable success. Dent Traumatol 18:157-180.

15) Watanabe Y, Mohri T, Takeyama M, Yamaki M, Okiji T, Saito C, Saito I (2010) Long-term observation of autotransplanted teeth with complete root formation in orthodontic patients. Am J Orthod Dentofacial Orthop 138:720-726.

16) Yoshino K, Kariya N, Namura D, Noji I, Mitsuhashi K, Kimura H, Fukuda A, Kikukawa I, Hayashi T, Yamazaki N (2012) A retrospective survey of autotransplantation of teeth in dental clinics. J Oral Rehabil 39:37-43.

\section{Correspondence:}

Dr. Tahir Yusuf Noorani

Conservative Dentistry Unit,

School of Dental Sciences,

Universiti Sains Malaysia,

16150 Kubang Kerian,

Kelantan, Malaysia

E-mail: dentaltahir@yahoo.com 